\title{
Support Infornation
}

\author{
for
}

\section{Template-Assisted Stereoselective Photocyclodimerization of 2- Anthracenecarboxylic Acid by Bispyridinio-Appended $\gamma$-Cyclodextrin}

\author{
Hiroshi Ikeda*, Takashi Nihei, and Akihiko Ueno \\ Department of Bioengineering, Graduate School of Bioscience and Biotechnology, \\ Tokyo Institute of Technology, \\ 4259-B-46 Nagatsuta-cho, Midori-ku, Yokohama 226-8501, Japan \\ E-mail: hikeda@bio.titech.ac.jp
}

\section{CONTENTS}

Figure S1 ${ }^{1} \mathrm{H}$ NMR spectrum of Py2(AE)- $\boldsymbol{P}$-CD $\ldots \ldots \ldots \ldots \ldots . . . . .62$

Figure S2 ${ }^{1} \mathrm{H}$ NMR spectrum of Py2(AD)- $\boldsymbol{\gamma}$-CD $\ldots \ldots \ldots \ldots \ldots . . . . . .6$

Figure S3 ${ }^{1} \mathrm{H}$ NMR spectrum of Py2(AC)- $\boldsymbol{\gamma}$-CD $\ldots \ldots \ldots \ldots \ldots . . . . .6$

Figure S4 ${ }^{1} \mathrm{H}$ NMR spectrum of Py2(AB)- $\boldsymbol{\gamma}$-CD $\ldots \ldots \ldots \ldots \ldots \ldots . . \ldots$ S5

Figure S5 ${ }^{1} \mathrm{H}$ NMR spectrum of Py- $\boldsymbol{P}$-CD $\quad \ldots \ldots \ldots \ldots \ldots \ldots \ldots . . . \ldots 6$

Assinments of ${ }^{1} \mathrm{H}$ NMR spectra of pyridinio-modified CDs $\cdots \cdots \cdots \quad$ S7 


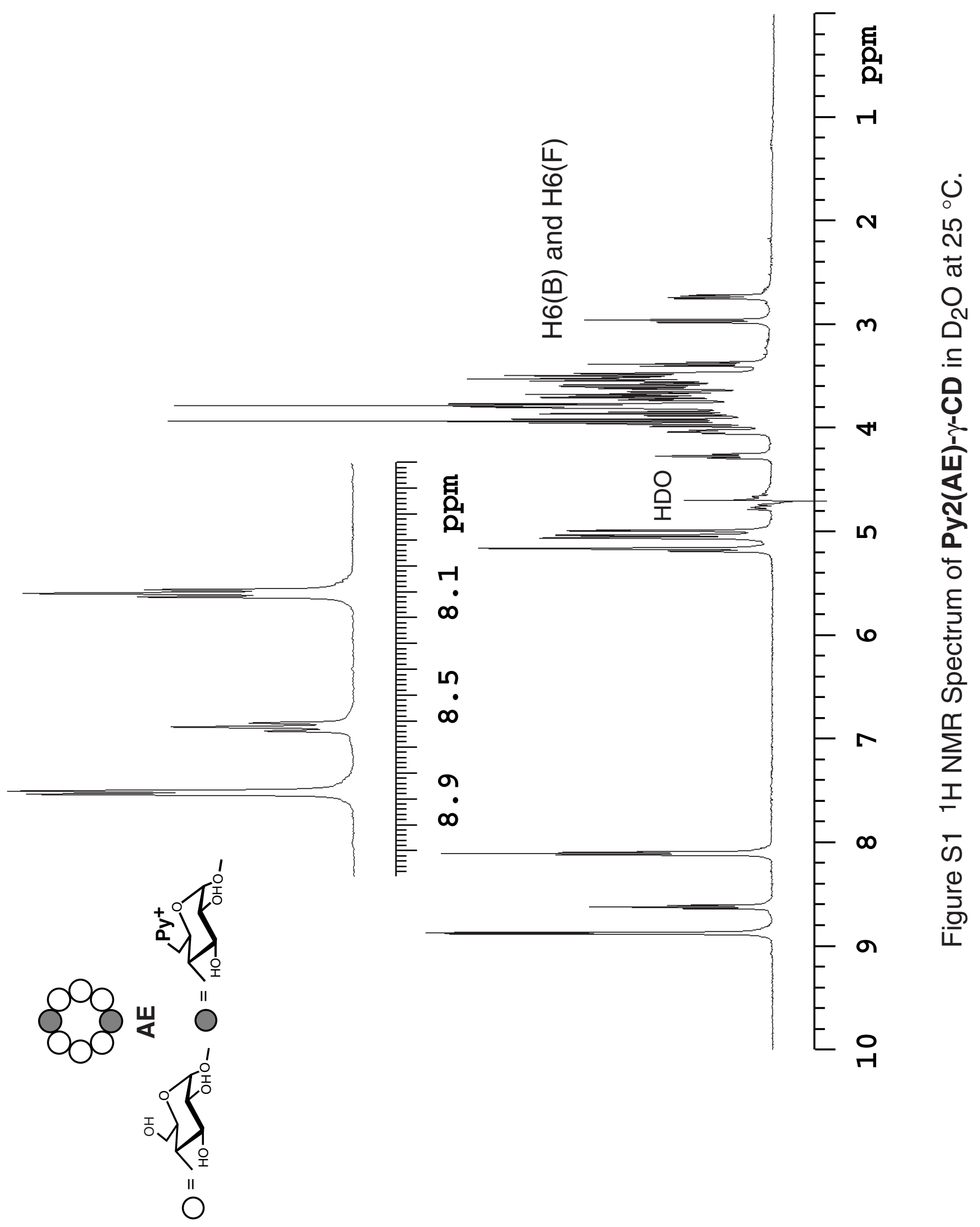




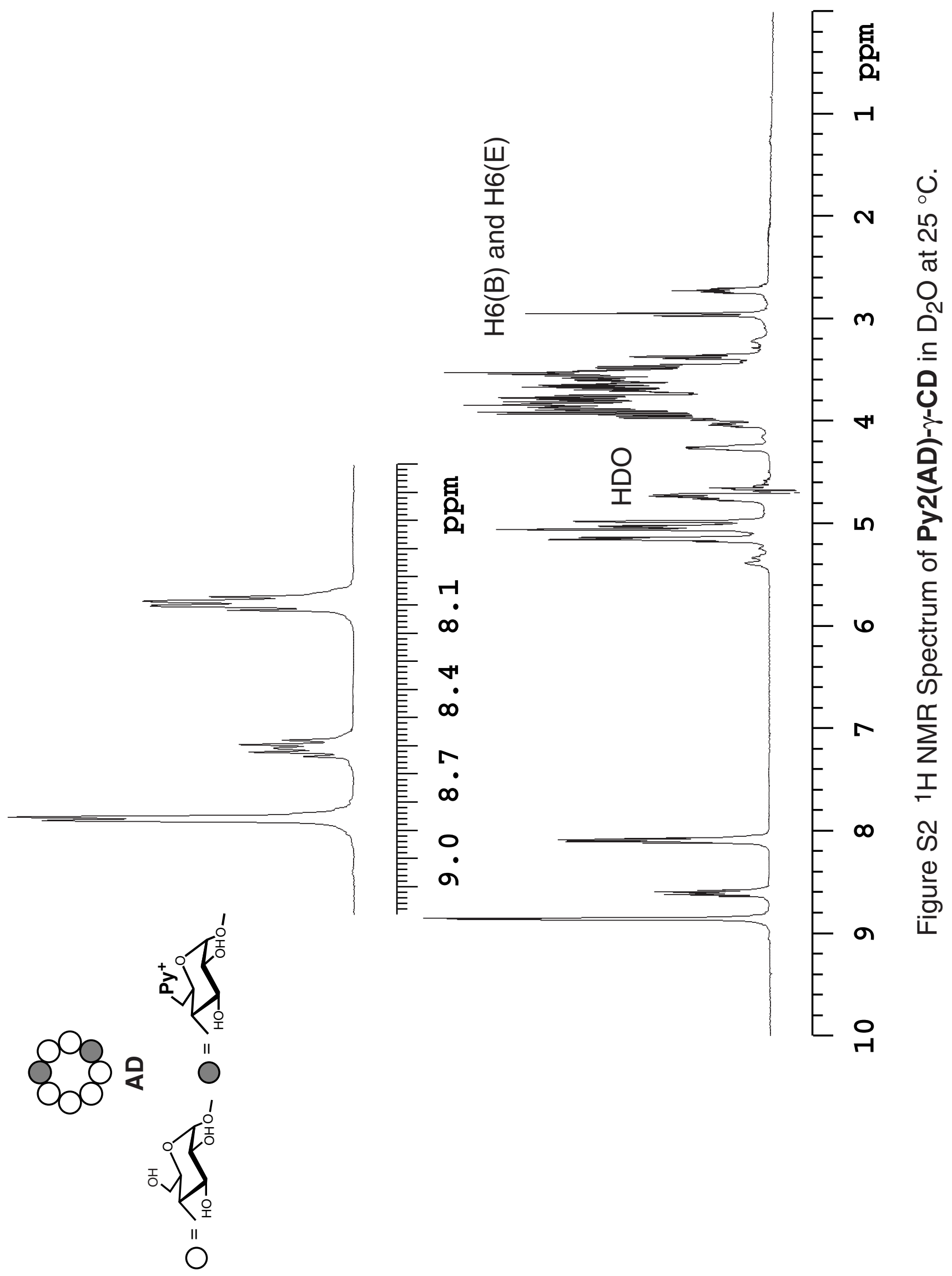




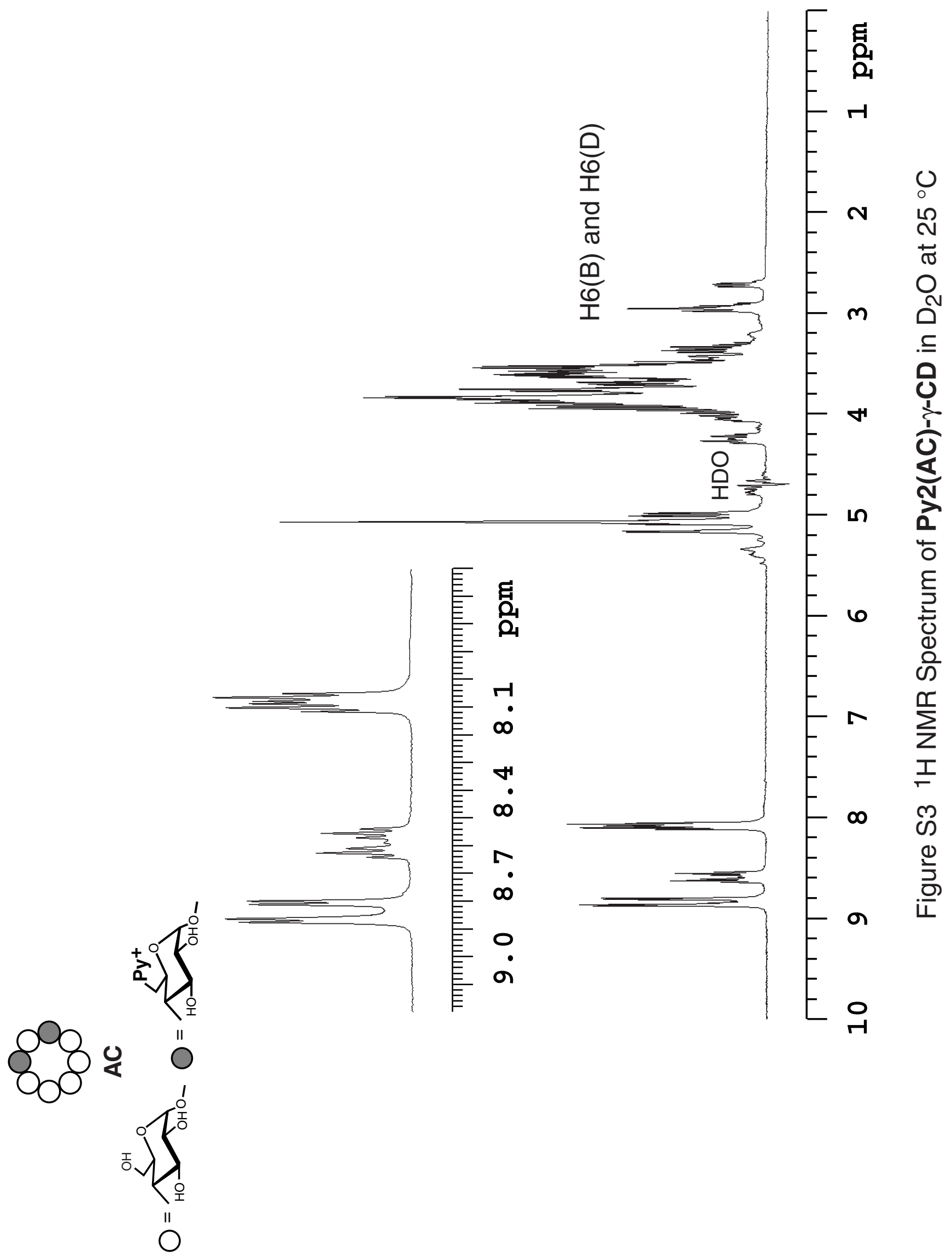




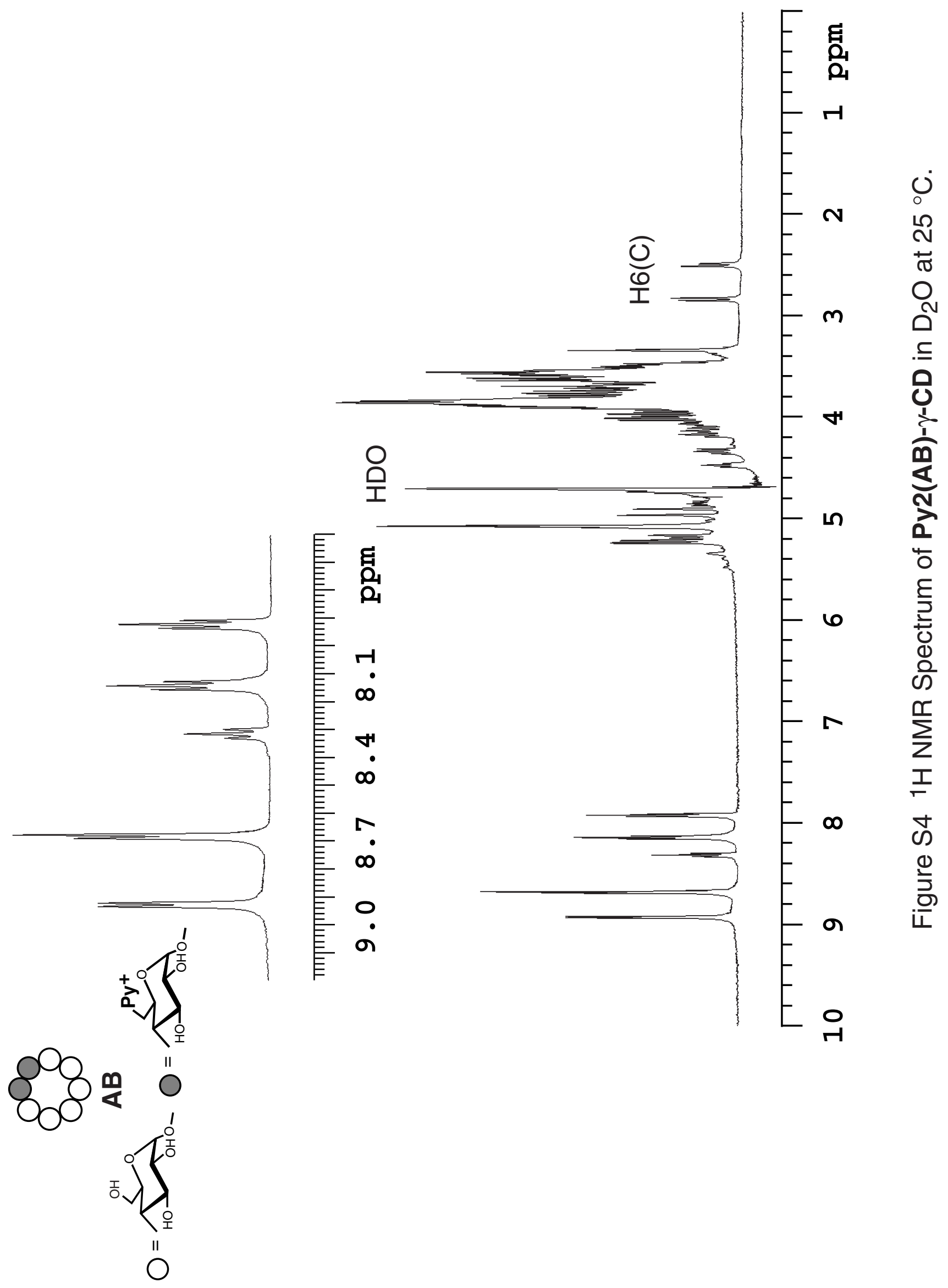




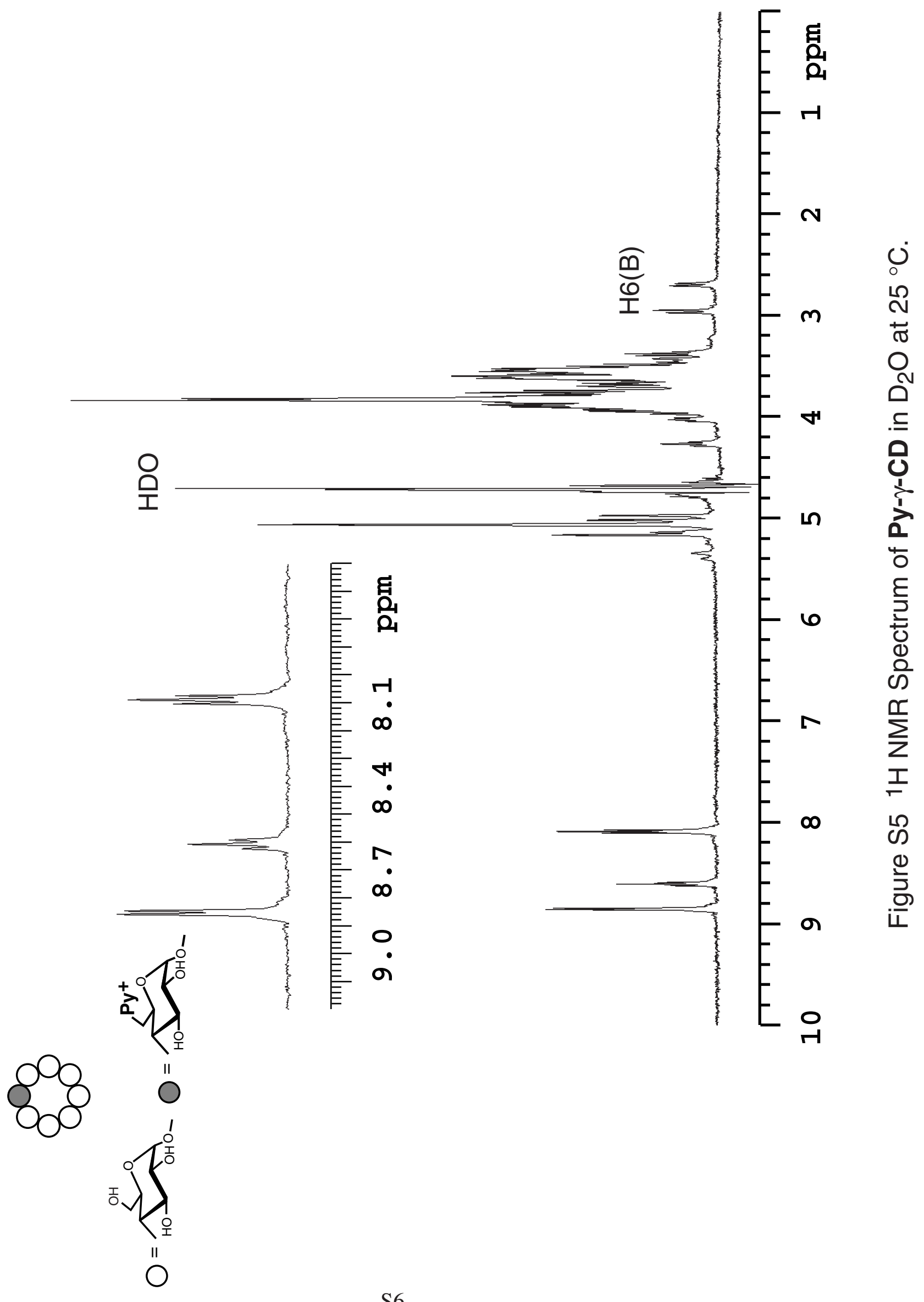




\section{Assinments of ${ }^{1} \mathrm{H}$ NMR spectra of pyridinio-modified CDs}

\section{$\operatorname{Py2}(\mathrm{AE})-\boldsymbol{\gamma}-\mathrm{CD}$}

${ }^{1} \mathrm{H}$ NMR (500 MHz, $\mathrm{D}_{2} \mathrm{O}$ ): $\delta=2.73(\mathrm{dd}, J=4.5,12.3 \mathrm{~Hz}, 2 \mathrm{H}, \mathrm{H}-6 \mathrm{~b}, \mathrm{H}-6 \mathrm{~b}), 2.97(\mathrm{dd}, J=2.2,12.3 \mathrm{~Hz}$, 2H, H-6a, H-6a), 4.99 (d, $J=3.9 \mathrm{~Hz}, 2 \mathrm{H}, \mathrm{H}-1), 5.03$ (d, $J=3.9 \mathrm{~Hz}, 2 \mathrm{H}, \mathrm{H}-1$ ), 5.06 (d, $J=4.2 \mathrm{~Hz}, 2 \mathrm{H}$, H-1), $5.16(\mathrm{~d}, J=3.9 \mathrm{~Hz}, 2 \mathrm{H}, \mathrm{H}-1), 8.10(\mathrm{dd}, J=7.8,6.8 \mathrm{~Hz}, 4 \mathrm{H}, \mathrm{Py}-3), 8.62(\mathrm{tt}, J=7.8,1.2 \mathrm{~Hz}, 2 \mathrm{H}$, Py-4), 8.87 (dd, $J=6.8,1.2 \mathrm{~Hz}, 4 \mathrm{H}, \mathrm{Py}-2$ ).

\section{Py2(AD)- $\gamma-C D$}

${ }^{1} \mathrm{H}$ NMR $\left(500 \mathrm{MHz}, \mathrm{D}_{2} \mathrm{O}\right): \delta=2.73(\mathrm{dd}, J=4.4,12.3 \mathrm{~Hz}, 1 \mathrm{H}, \mathrm{H}-6 \mathrm{~b}), 2.74(\mathrm{dd}, J=4.8,12.3 \mathrm{~Hz}, 1 \mathrm{H}, \mathrm{H}-$ 6b), 2.97 (bd, $J=12.3 \mathrm{~Hz}, 2 \mathrm{H}, \mathrm{H}-6 \mathrm{a}$ ), 4.99 (bd, $J=3.9 \mathrm{~Hz}, 2 \mathrm{H}, \mathrm{H}-1$ ), 5.03 (d, $J=3.9 \mathrm{~Hz}, 1 \mathrm{H}, \mathrm{H}-1$ ), 5.04 (d, $J=3.7 \mathrm{~Hz}, 1 \mathrm{H}, \mathrm{H}-1), 5.06$ (d, $J=3.9 \mathrm{~Hz}, 1 \mathrm{H}, \mathrm{H}-1), 5.07$ (d, $J=3.9 \mathrm{~Hz}, 1 \mathrm{H}, \mathrm{H}-1), 5.15$ (d, $J=3.9 \mathrm{~Hz}$, 1H, H-1), 5.16 (d, $J=3.9$ Hz, 1H, H-1), 8.09 (t, $J=7.2 \mathrm{~Hz}, 2 \mathrm{H}$, Py-3), 8.11 (t, $J=7.2 \mathrm{~Hz}, 2 \mathrm{H}, \mathrm{Py}-3$ ), 8.60 (t, $J=7.2 \mathrm{~Hz}, 1 \mathrm{H}, \mathrm{Py}-4), 8.63$ (t, $J=7.2 \mathrm{~Hz}, 1 \mathrm{H}, \mathrm{Py}-4), 8.87$ (d, $J=7.2 \mathrm{~Hz}, 4 \mathrm{H}, \mathrm{Py}-2)$.

\section{Py2(AC)- $\gamma-C D$}

${ }^{1} \mathrm{H}$ NMR $\left(500 \mathrm{MHz}, \mathrm{D}_{2} \mathrm{O}\right): \delta=2.72(\mathrm{dd}, J=4.3,12.3 \mathrm{~Hz}, 1 \mathrm{H}, \mathrm{H}-6 \mathrm{~b}), 2.92(\mathrm{dd}, J=6.4,12.3 \mathrm{~Hz}, 1 \mathrm{H}$, H-6b), 2.97 (dd, $J=2.2,12.3 \mathrm{~Hz}, 1 \mathrm{H}, \mathrm{H}-6 \mathrm{a}), 2.97$ (dd, $J=2.2,12.3 \mathrm{~Hz}, 1 \mathrm{H}, \mathrm{H}-6 \mathrm{a}$ ), 4.98 (d, $J=3.9 \mathrm{~Hz}$, 1H, H-1), 5.01 (d, $J=3.9 \mathrm{~Hz}, 1 \mathrm{H}, \mathrm{H}-1), 5.07$ (d, $J=3.9 \mathrm{~Hz}, 3 \mathrm{H}, \mathrm{H}-1), 5.08$ (d, $J=3.9 \mathrm{~Hz}, 1 \mathrm{H}, \mathrm{H}-1)$, $5.09(\mathrm{~d}, J=3.9 \mathrm{~Hz}, 1 \mathrm{H}, \mathrm{H}-1), 5.17(\mathrm{~d}, J=3.9 \mathrm{~Hz}, 1 \mathrm{H}, \mathrm{H}-1), 8.07$ (dd, $J=7.8,6.8 \mathrm{~Hz}, 2 \mathrm{H}, \mathrm{Py}-3), 8.10$ (dd, $J=7.8,6.8 \mathrm{~Hz}, 2 \mathrm{H}$, Py-3), 8.55 (tt, $J=7.8,1.2 \mathrm{~Hz}, 1 \mathrm{H}, \mathrm{Py}-4), 8.63$ (tt, $J=7.8,1.2 \mathrm{~Hz}, 1 \mathrm{H}, \mathrm{Py}-4$ ), $8.81(\mathrm{dd}, J=6.8,1.2 \mathrm{~Hz}, 2 \mathrm{H}, \mathrm{Py}-2), 8.87(\mathrm{dd}, J=6.8,1.2 \mathrm{~Hz}, 2 \mathrm{H}, \mathrm{Py}-2)$.

\section{$\operatorname{Py} 2(\mathrm{AB})-\boldsymbol{\gamma}-\mathrm{CD}$}

${ }^{1} \mathrm{H}$ NMR $\left(500 \mathrm{MHz}, \mathrm{D}_{2} \mathrm{O}\right): \delta=2.50(\mathrm{dd}, J=4.0,12.3 \mathrm{~Hz}, 1 \mathrm{H}, \mathrm{H}-6 \mathrm{~b}), 2.84(\mathrm{bd}, J=12.3 \mathrm{~Hz}, 1 \mathrm{H}, \mathrm{H}-$ 6a), 4.90 (d, $J=3.4 \mathrm{~Hz}, 1 \mathrm{H}, \mathrm{H}-1), 4.97$ (d, $J=3.9 \mathrm{~Hz}, 1 \mathrm{H}, \mathrm{H}-1), 5.07$ (d, $J=3.9 \mathrm{~Hz}, 2 \mathrm{H}, \mathrm{H}-1), 5.09$ (d, $J=3.9 \mathrm{~Hz}, 2 \mathrm{H}, \mathrm{H}-1), 5.22(\mathrm{~d}, J=3.9 \mathrm{~Hz}, 1 \mathrm{H}, \mathrm{H}-1), 5.24(\mathrm{~d}, J=3.9 \mathrm{~Hz}, 1 \mathrm{H}, \mathrm{H}-1), 7.92(\mathrm{dd}, J=7.8,6.8$ $\mathrm{Hz}, 2 \mathrm{H}, \mathrm{Py}-3), 8.14$ (dd, $J=7.8,6.8 \mathrm{~Hz}, 2 \mathrm{H}, \mathrm{Py}-3), 8.31$ (tt, $J=7.8,1.2 \mathrm{~Hz}, 1 \mathrm{H}, \mathrm{Py}-4), 8.68$ (tt, $J=7.8$, $1.2 \mathrm{~Hz}, 1 \mathrm{H}, \mathrm{Py}-4), 8.68$ (dd, $J=6.8,1.2 \mathrm{~Hz}, 2 \mathrm{H}, \mathrm{Py}-2), 8.93$ (dd, $J=6.8,1.2 \mathrm{~Hz}, 2 \mathrm{H}, \mathrm{Py}-2)$. 


\section{Py- $\boldsymbol{\gamma}-\mathrm{CD}$}

${ }^{1} \mathrm{H}$ NMR $\left(500 \mathrm{MHz}, \mathrm{D}_{2} \mathrm{O}\right): \delta=2.69(\mathrm{dd}, J=4.2,12.6 \mathrm{~Hz}, 1 \mathrm{H}, \mathrm{H}-6 \mathrm{~b}), 2.96(\mathrm{dd}, J=2.0,12.6 \mathrm{~Hz}, 1 \mathrm{H}, \mathrm{H}-$ 6a), 4.97 (d, $J=3.9 \mathrm{~Hz}, 1 \mathrm{H}, \mathrm{H}-1), 5.02(\mathrm{~d}, J=3.7 \mathrm{~Hz}, 1 \mathrm{H}, \mathrm{H}-1), 5.06$ (bd, $J=3.9 \mathrm{~Hz}, 5 \mathrm{H}, \mathrm{H}-1), 5.16$ (d, $J=4.1 \mathrm{~Hz}, 1 \mathrm{H}, \mathrm{H}-1), 8.09(\mathrm{t}, J=7.6 \mathrm{~Hz}, 2 \mathrm{H}, \mathrm{Py}-3), 8.61(\mathrm{t}, J=7.6 \mathrm{~Hz}, 1 \mathrm{H}, \mathrm{Py}-4), 8.85(\mathrm{~d}, J=7.6 \mathrm{~Hz}$, 2H, Py-2). 